\title{
Narrowing the interface between sample preparation and electrochemistry: Trace-level determination of emerging pollutant in water samples after in situ microextraction and electroanalysis using a new cell configuration
}

\author{
Jéssica Vita Gabbana a, Luiz Henrique de Oliveira ${ }^{\text {a }}$, Guilherme Caneppele Paveglio ${ }^{\text {b }}$, \\ Magno Aparecido Gonçalves Trindade ${ }^{\text {a, c, * }}$ \\ a Faculdade de Ciências Exatas e Tecnologia, Universidade Federal da Grande Dourados, Rodovia Dourados-Itahum, km 12, Dourados, MS, 79804-970, Brazil \\ b State University of Mato Grosso do Sul, Rodovia Dourados-Itahum, km 12, Dourados, MS, 79804-970, Brazil \\ ${ }^{\mathrm{c}}$ Unesp, National Institute for Alternative Technologies of Detection, Toxicological Evaluation and Removal of Micropollutants and Radioactives (INCT- \\ DATREM), Institute of Chemistry, P.O. Box 355, 14800-900, Araraquara, SP, Brazil
}

\section{A R T I C L E I N F O}

\section{Article history:}

Received 13 February 2018

Received in revised form 16 April 2018

Accepted 19 April 2018

Available online 21 April 2018

\section{Keywords:}

In situ microextraction

Contaminant of emerging concern

Electroanalysis at trace-level

Residual drugs determination

\begin{abstract}
A B S T R A C T
In this work, a new cell configuration that enables the in situ integration of sample preparation and electrochemical detection prior analysis at trace-level is described. To perform the microextraction, a green chemistry extractor solvent, so called deep eutectic solvent (DES) was used, which allowed the pre-concentration of ciprofloxacin (CIPRO) from water samples. The particular characteristics of the featured device include: reduction of the number of steps involved in conventional liquid-liquid microextraction, processing the pre-concentration of target analyte in a small drop that can be directly decanted onto the electrode surface and further electroanalysis at a single time in order to achieve the required trace-level quantity. After the optimization of parameters inherent to the system, with the aid of chemometric approach, the proposed device enabled fast electroanalysis of CIPRO at concentration levels down to $5.00 \mathrm{nmol} \mathrm{L}^{-1}$ with suitable accuracy, since the recovery values were ranged between 71 and $93 \%$.
\end{abstract}

() 2018 Elsevier Ltd. All rights reserved.

\section{Introduction}

Dispersive Liquid-liquid microextraction (DLLME) has received considerable attention as a type of miniaturised extraction technique that offers simplicity, ease of handling, low sample consumption, high speed, reduced processing costs and time, the requirement for negligible volumes of extractor solvent, and reduced residue generation [1-3]. Progress has also been made in terms of its ability to pre-concentrate target analytes from very dilute aqueous samples without using a large volume of toxic organic solvents, which makes the process environmentally friendly. DLLME was initially proposed by Assadi and coworkers [4],

\footnotetext{
* Corresponding author. Faculdade de Ciências Exatas e Tecnologia, Universidade Federal da Grande Dourados, Rodovia Dourados-Itahum, km 12, Dourados, MS 79804, Brazil.

E-mail addresses: magnotr@gmail.com, magnotrindade@urge.edu.br (M.A.G. Trindade).
}

and the procedure was based on the dispersion of the extractor solvent into the aqueous sample, usually assisted by a disperser agent. Although the original technique involved toxic high-density extractor solvents such as chlorobenzene, chloroform or carbon tetrachloride, LLME has enabled the use of less toxic solvents in developing new applications for enhanced-solvent extractions [5-7]. Since the introduction of the so-called type III deep eutectic solvents (DESs) in 2004 [8], a large field of application has been opened up in analytical chemistry. These solvents are of particular interest due to their highly biodegradable properties [9], which are relevant in association with studies employing alternative and selective green chemistry solvents within the framework of DLLME [10,11].

Regarding electrochemical techniques, the main advantage is associated with low-cost instrumentation and a variety of voltammetric techniques being available, thus allowing the miniaturization of electrochemical device (for analysis in small volumes) without loses the sensitivity [12,13]. Despite the advantages, a 
potential drawback in residual analyte determination is related to the intense background and/or interference from matrix concomitants that can strongly affect the accuracy of measurements of the electrochemical signal $[13,14]$ '. In such scenarios, sample preparation should be considered, prior to the actual analysis, for at least two reasons. Firstly, the sample should be processed to eliminate any potential matrix effect that could compromise the accurate measurement of the electrochemical signal, and secondly, adjustments of sample volume should be carried out to pre-concentrate the target analyte in order to achieve the desired quantitation limit $[15,16]$. In addition to this, without the pre-concentration step, the most sensitive electroanalytical techniques, square-wave voltammetry (SWV) and differential pulse voltammetry (DPV), can only detect organic compounds in the range of $10^{-7}-10^{-6} \mathrm{~mol} \mathrm{~L}^{-1}$ [12-14]. Moreover, conventional electroanalysis usually involves classical cell configurations, which may further dilute the analyte in the electrochemical cell [17].

Generally, to ensure that the electroanalytical techniques operate with sufficient sensitivity and meet the quantitation limit required for trace analyses, a pre-concentration step of the analytes onto the working electrode surface, followed by stripping voltammetric analysis, is performed $[12,18]$. However, the electrochemical pre-concentration step has a disadvantage during the analysis of polar organic compounds, as the generated products can foul the electrode surface, thus hindering the electron transfer process [19-21]. In such cases, the removal of surface fouling between each measurement is difficult, which, in turn, hinders the ability to reestablish the electrode-surface-activity [20].

Trindade and coworkers have used some fluoroquinolones (FQs) as electrochemical probes for simultaneous determination [22-24], and showed that the electro-oxidation on carbon based electrodes occurs at potential range between 0.75 and $1.14 \mathrm{~V}$. The overlapping peaks observed and the limited selectivity of electroanalytical techniques did not allow simultaneous detection. In addition, it was also showed that the quantification at trace-levels is a shortcoming, since the voltammetric profile was almost undistinguishable and/or the peak current could not be accurately measured [22]. To overcome these drawbacks, it was used a preconcentration based on a combination of liquid-liquid microextraction and mathematical processing (baseline-corrected second-order derivative), in which this process contains several steps. Herein, a new cell configuration for in situ extraction and preconcentration (based on liquid-liquid microextraction) and further electroanalysis at the trace-level is proposed. To evaluate the performance of proposed electrochemical device, ciprofloxacin (CIPRO) a popular antibiotic member of fluoroquinolone class, was used as a model analyte because of its constant appearance in the list of contaminant [25-27] as emerging pollutants in water samples.

\section{Experimental section}

\subsection{Chemicals, solutions, and samples}

The ciprofloxacin (CIPRO) was purchased from Sigma Aldrich and used as received. The standard stock solutions were prepared at the concentration of $10.0 \mathrm{mmol} \mathrm{L}^{-1}$ by dissolving their powder in $0.5 \%$ of acetic acid (Sigma-Aldrich) and further diluting solutions with ultrapure water. The working solutions, in the concentration range from 5.00 to $10.0 \mathrm{nmol} \mathrm{L}^{-1}$, were prepared daily by diluting the stock standard solutions. The supporting electrolyte (at a concentration of $40 \mathrm{mmol} \mathrm{L}^{-1}$ ) was a buffer Britton-Robinson (B-R) solution and was prepared by a mixture of acetic acid, phosphoric acid, and boric acid (all purchased from Sigma-Aldrich ${ }^{\circledR}$, São Paulo, Brazil), and further adjustment of the required $\mathrm{pH}$ by using ammonium hydroxide (Sigma-Aldrich) at a concentration of $1.0 \mathrm{~mol} \mathrm{~L}^{-1}$. All other reagents and solvents used in this study were of analytical grade, purchased from Sigma-Aldrich and used as received. The extractor solvents tested include two deep eutectic solvent (DES): the choline chloride:malonic acid (ChCl:MA at 1:1, molar ratio) and choline chloride:p-toluenesulfonic acid ( $\mathrm{ChCl}: \mathrm{TsOH}, 1: 1$ ), both synthesised as shown in the experimental section (Supplementary material).

\subsection{Instrumentation and electrochemical device design}

For electrochemical experiments, a potentiostat/galvanostat PGSTAT 204 (Metrohm Autolab) was used, controlled by the software Nova 1.11. The $\mathrm{pH}$ measurements were performed using combined glass electrode (Hanna, model HI 1131 B, Texas, United States) connected to a digital pH-meter (Hanna, model HI 3221). The ultrapure water $(R \geq 18.2 \mathrm{M} \Omega \mathrm{cm})$ used for the preparation of the working solutions was obtained in an OS 10 LTXE reverse osmosis (Gehaka) and further water purification system (Thermo Scientific, model Barnstead Smart2Pure). The electrochemical cell, adapted to the extraction, is shown in Fig. 1. The electrochemical system was composed by a jacketed electrochemical cell (a), with the maximum capacity of $30 \mathrm{~mL}$ of sample or electrolyte reservoir. The conventional three-electrode arrangement was composed by a

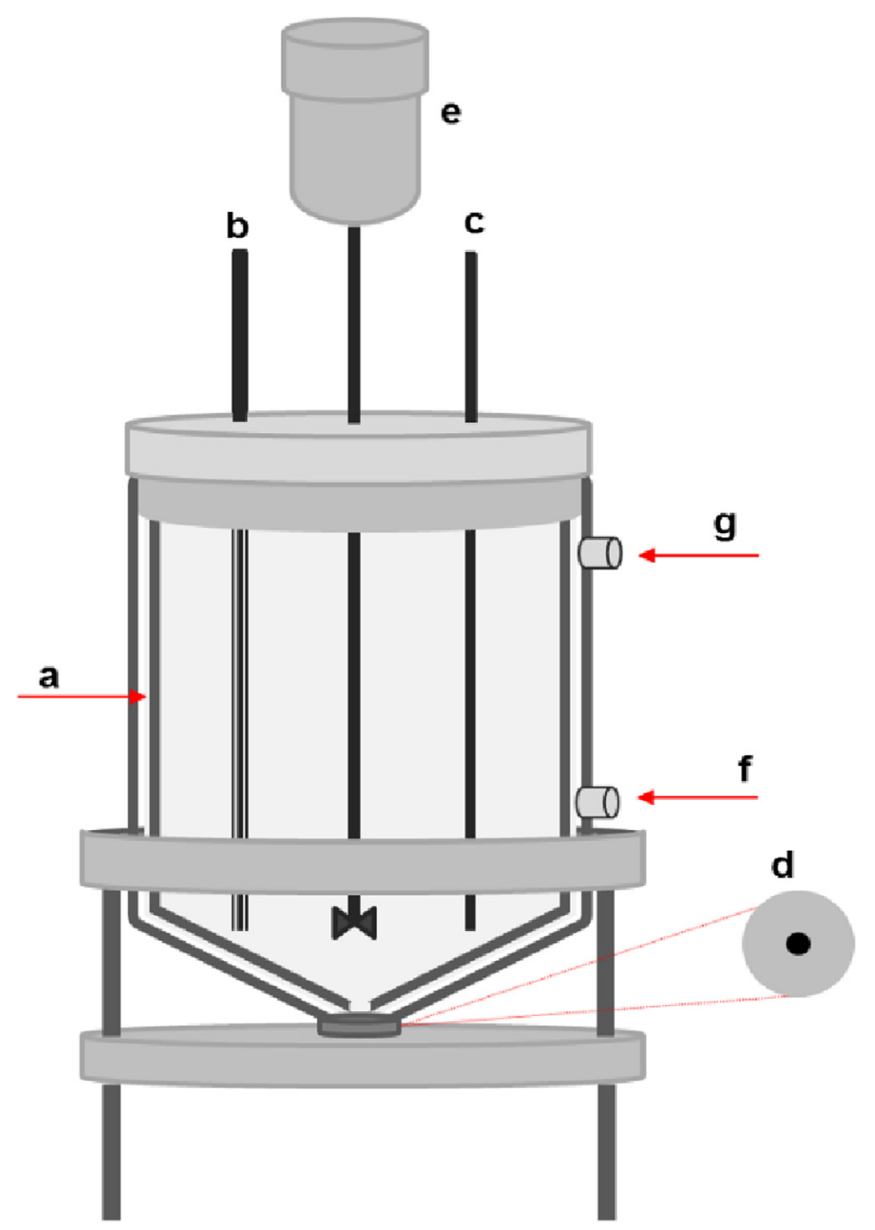

Fig. 1. Schematic representation of the new electrochemical cell configuration. (a) Jacketed electrochemical cell (capacity of $30 \mathrm{~mL}$ of sample or electrolyte reservoir), (b) $\mathrm{Ag} / \mathrm{AgCl}(\mathrm{KCl}$ sat. $)$ as the reference electrode, (c) platinum wire as the auxiliary electrode and (d) working electrode: a disc of glassy carbon ( $2 \mathrm{~mm}$ of geometric area), (e) printer engine, (f) inlet and (g) outlet for cooling water. 
$\mathrm{Ag} / \mathrm{AgCl}_{\text {(KClsat.) }}$ as the reference electrode (b), a platinum wire as the auxiliary electrode (c) and a disc of glassy carbon (GC, $2 \mathrm{~mm}$ of geometric area) as the working electrode (d). The configuration also contain a printer engine (as the stirring rod mechanical stirrer, $\mathbf{e}$ ) on top of the cell and connected to an adjustable (1.5-12 V) DC power supply for control the stirring speed, an inlet for cooling water (f) and outlet for cooling water $(\mathbf{g})$. The complete schematic representation of the new cell configuration can be seen in Fig. S-2 (Supplementary material).

\subsection{Sample preparation and electroanalysis}

Tap water samples were collected from the water supply network of the Laboratory of Analytical Chemistry at the Federal University of Grande Dourados (Dourados, MS, Brazil) and spiked with CIPRO at two-concentration levels (5.00 and $10.0 \mathrm{nmol} \mathrm{L}^{-1}$ ). The target-spiked samples were prepared by adding the required amount of the CIPRO working solution into the tap water samples together with the supporting electrolyte solution (B-R buffer), followed by manual or vortex shaking for $5 \mathrm{~min}$. The spiked tap water samples were stored in glass bottles, conserved at $4.0^{\circ} \mathrm{C}$ and analysed without any pre-treatment or filtration.

To carry out the extraction procedure, $25 \mathrm{~mL}$ of spiked tap water samples and an appropriate mass of target extractor (DES, ChCl:MA at $1: 1$, molar ratio) were introduced into electrochemical cell configuration. Afterwards, the sample was shaken using the mechanical flow (Fig. 1e) under previously varied times (between 3 and $15 \mathrm{~min}$ ), which first allowed dispersion and then separation between the aqueous phase and the high-density solvent. After this step, the aquarium pump (Fig. S-2k) was turned on, allowing the recirculation of ice water into the jacketed electrochemical cell to provide the decantation of enriched residues from the $\mathrm{ChCl}: \mathrm{MA}$ $(1: 1$, molar ratio) phase in the GCE surface. The voltammetric measurements were performed for a target-enriched solution (with an appropriate electrolytic medium). Unless otherwise indicated, all measurements were performed in triplicate and standard deviations were also calculated. After each electrochemical measurement, to reestablish the electrode-surface-activity the solution was mechanically stirred (during 120 s), which allowed a turbulent flow to be applied directly to the GCE surface.

\subsection{Data treatment}

After registering the original DP voltammograms, the signal transformation based on the baseline-corrected second-order derivative was performed using the procedure highlighted in a previous work [22]. The Originlab ${ }^{\circledR}$ (version 9.0) software was used, in which the process includes the following steps: baseline-correction followed by the insertion of the second-order derivative mathematical function and finished with the baseline-correction again. This sequence was a key factor to provide more accurate measurement as well as the intensification of target CIPRO peak.

Data treatment using $2^{3}$ factorial design was also performed to evaluate the performance of the proposed device. The factorial design was based on the spreadsheets for experimental design calculations (software Excel ${ }^{\circledR}$, Microsoft Office, 2003) proposed by Teófilo and Ferreira [28]. The variables and levels used includes: ChCl:MA (1:1, molar ratio) mass (40-160 mg), stirring time (5-10 $\mathrm{min})$ and cooling time (5-10 $\mathrm{min})$, taking into consideration the low (-1) and high (1) level. To compare the magnitude and statistical significance of each main effect and its interactions, the data were available in the Pareto chart and normal probability plot. For statistical treatment using the Doehlert matrix (with a central point), at 95\% confidence level, it was used the StatSoft Statistica ${ }^{\circledR}$ 10.0 software package (Statsoft, Tulsa, U.S.).

\section{Results and discussion}

\subsection{Voltammetric study to optimise the experimental conditions}

Before evaluation the parameters inherent to the performance of the proposed device (e.g., in extract and pre-concentrate target CIPRO), the new cell configuration was used to obtain some voltammograms in a classical mode (i.e., absence of extractor solvent and pre-concentration step). Fig. 2 show the voltammograms recorded using differential pulse voltammetry (DPV) and squarewave voltammetry (SWV), in which the working conditions were chosen after studying the aqueous supporting electrolyte and its performance, as shown in Fig. S-3 (Supplementary material). In such cases, both techniques generate an intense electrochemical signal, with peak potential at $1.12 \mathrm{~V}$ (DPV) and $1.16 \mathrm{~V}$ (SWV), respectively. From an analytical viewpoint, the DPV was chosen for further measurements, because its best performance allowed the accurate measurement of the CIPRO (at $0.62 \mu \mathrm{mol} \mathrm{L}^{-1}$ ) oxidation peak. However, as demonstrated in previous works [22,23], the major challenge in electro-oxidation of the FQ piperazinyl group at the 7-position is the difficulty in preventing the easy fouling of the electrode surface. To re-establish the electrode-surface-activity and improve voltammetric analysis, the use of surfactants and polishing the electrode surface between measurements was necessary. The procedure implies that, for the electroanalysis of CIPRO using the classical electrochemical system, this may last for several minutes or hours. Then, in addition to the evaluation of the parameters inherent to the DPV technique (Table S- 1 , Supplementary material), we also deal with the cleaning of the electrode surface to remove adsorbed species and obtain an active electrode surface able to generate reproducible results.

\subsection{In situ cleaning of the electrode surface}

The cleaning of the electrode surface was performed by, in situ, mechanically stirring the supporting electrolyte solution, between each measurement, to direct a turbulent flow up to the electrode

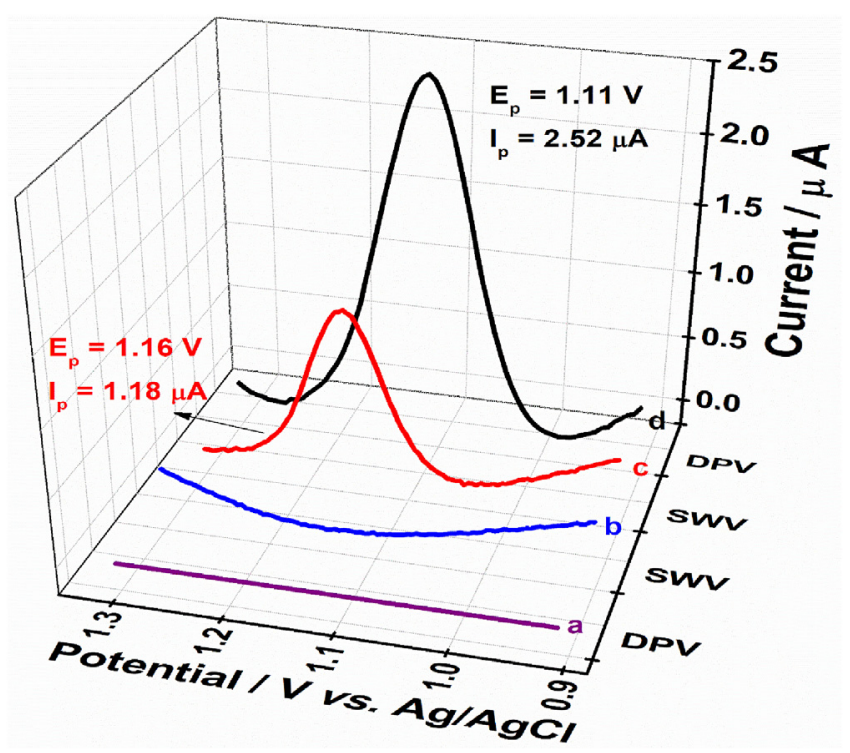

Fig. 2. Voltammograms (with base-line correction) recorded in absence (a) DPV and (b) SWV and in the presence (c) SWV and (d) DPV of CIPRO at $0.62 \mu \mathrm{mol} \mathrm{L}^{-1}$. Experimental conditions: $40 \mathrm{mmol} \mathrm{L}^{-1}$ Britton-Robinson (B-R) buffer at $\mathrm{pH} 3.0$ as supporting electrolyte. Parameters: DPV pulse amplitude $\left(\mathrm{E}_{\mathrm{sW}}\right)=100 \mathrm{mV}$; step potential $\left(\Delta \mathrm{E}_{\mathrm{s}}\right)=6 \mathrm{mV}$; Pulse time $=0.025 \mathrm{~s}$; scan rate $(U)=20 \mathrm{mV} \mathrm{s}^{-1}$. SWV conditions: $\mathrm{E}_{\mathrm{sw}}=25 \mathrm{mV} ; \Delta \mathrm{Es}=4 \mathrm{mV}$ and frequency $(f)=10 \mathrm{~Hz}$. 
surface (Fig. 3). The voltammetric performance shown in Fig. 3 (voltammograms b and c) reveals a lowering of the peak current after successive measurements in the absence of mechanical stirring. This is a consequence of the formation of passive films via electrochemically generated products on the GCE surface, which in turn, blocks the electrode activity. However, after $60 \mathrm{~s}$ of in situ mechanical stirring (Fig. 3, voltammogram d), the partial removal of electrogenerated products can be seen, because the peak current was only $11 \%$ lower than that obtained with the polished electrode surface (Fig. 3, voltammogram a). Indeed, our approach based on a homemade stirring rod (Fig. 1e) worked properly and can be used to avoid electrode fouling by removing the deposited product layer from its surface. This can be proven by studying the stirring time effects (Fig. S-4, Supplementary material), where it was shown that $120 \mathrm{~s}$ (Fig. S-4, voltammogram d) of stirring of the electrolyte supporting solution was enough to re-establish the electrodesurface-activity. It is also worth noting that the loss in the peak current intensity was only $2.07 \%$ and $3.45 \%$ for 120 and 180 s (Fig. S4) voltammograms c and d, respectively. In such conditions, the performance under prolonged operative time, of up to 30 consecutive measurements, also did not show any significant peak current fluctuations. Thus, $120 \mathrm{~s}$ was shown to be useful for the stirring step and was used for further measurements, where the total time between the electrode surface regeneration and the acquisition of the new voltammograms was no longer than $180 \mathrm{~s}$.

\subsection{Analytical curve obtained in absence of pre-concentration}

Under the optimised experimental and instrumental conditions (Figs. S-3 and S- 4 as well as Table S-1, Supplementary material), the signals derived from the detection of CIPRO after increasing the concentration range between 0.15 and $1.50 \mu \mathrm{mol} \mathrm{L}^{-1}$ were evaluated. From the typical calibration curve (Fig. S-5, Supplementary material), it can be seen that the proposed approach is useful for quantitative analysis in a linear range of $0.15-1.50 \mu \mathrm{mol} \mathrm{L}^{-1}$, with a

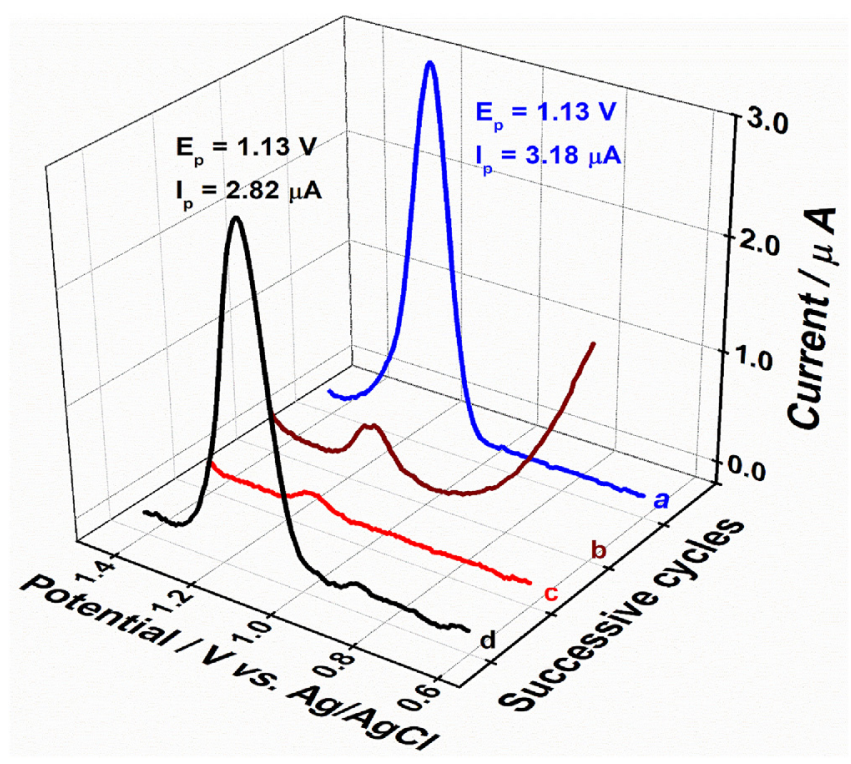

Fig. 3. DP voltammograms (with base-line correction) for electro-oxidation of CIPRO $\left(1.25 \mu \mathrm{mol} \mathrm{L}^{-1}\right)$ on the GCE and the using B-R buffer at $40 \mathrm{mmol} \mathrm{L}^{-1}(\mathrm{pH} 3.0)$ as supporting electrolyte solution. (a) Measurement on the clean GCE surface, (b) and (c) successive measurements without electrode surface clean between each experiments (absence of mechanical stirring), (d) measurement after $60 \mathrm{~s}$ of in situ mechanically stirring of the supporting electrolyte solution. Optimised conditions are shown in Table S-1 (Supplementary material). coefficient of determination $\left(R^{2}\right)$ greater than $0.99(n=7)$. The quantitative parameter is outlined in Table S-2 (Supplementary material), showing poor detectability when the newly developed approach is used as a classical electrochemical application (i.e., in absence of in situ pre-concentration). The statistical treatment $3 \times \mathbf{S d} / \mathbf{m}$ and $10 \times \mathbf{S d} / \mathbf{m}$ was used to calculate the limit of detection (LoD). In this, the Sd corresponds to the standard deviation for peak current values (at the same $\mathbf{E}_{\mathbf{p}}$ for CIPRO oxidation) measured from the register of ten voltammograms of the blank and $\boldsymbol{m}$ is the slope of the analytical curve. Even though the estimated value for LD was $0.12 \mu \mathrm{mol} \mathrm{L}^{-1}$ (Table S-2, Supplementary material), it still does not meet the requirements of the trace-level analysis, such as raised in previous works $[22,29]$. Thus, the following section deals with the in situ pre-concentration step and the optimization of parameters affecting its performance in order to improve de quantitative analysis of target analyte.

\subsection{Evaluation of the device for in situ microextraction}

To make the newly developed electrochemical device useful for trace-level analysis, we conducted a set of experiments in which pre-concentration using in situ microextraction was compared with the results acquired using direct electrochemical pre-concentration (Fig. 4). First, Fig. 4A shows the original DP voltammograms obtained under the previously optimised conditions by measurements of CIPRO at $10.0 \mathrm{nmol} \mathrm{L}^{-1}$ in spiked tap water samples and the absence of any mathematical process. At the same time, Fig. 4B shows the DP voltammograms after signal transformation using the baseline-corrected second-order derivative approach (as experimental section 2.4). The voltammograms registered for the blank (Fig. 4, voltammogram a) and the direct detection of CIPRO (at $10.0 \mathrm{nmol} \mathrm{L}^{-1}$ ) in spiked tap water samples in the absence of preconcentration (Fig. 4, voltammogram b) are characterised for the absence of any Faradaic process at the scanned potential range. Likewise, the detection using typical electrochemical preconcentration (at $0.4 \mathrm{~V}$ for $200 \mathrm{~s}$, Fig. 4, voltammogram c) of the sample (as listed in curve b) did not show any Faradaic process. Meanwhile, the voltammogram illustrated in Fig. 4 (curve e) showed that applying the pre-concentration step (with $120 \mathrm{mg}$ of $\mathrm{ChCl}$ :MA at 1:1, molar ratio) as extractor solvent) by using a target proposed device, enabled the extraction of CIPRO (at $10.0 \mathrm{nmol} \mathrm{L}^{-1}$ ) from the spiked tap water sample; the electroanalysis generates two partially distinguishable peaks at a potential of 1.13 and $1.39 \mathrm{~V}$ vs. $\mathrm{Ag} / \mathrm{AgCl}\left(\mathrm{KCl}_{\text {Sat. }}\right)$. Fig. 4 (voltammogram d) reveals the reliability of these results, where there is no detectable peak in the DP voltammogram registered after performing the same preconcentration process as for the blank sample.

It is worth mentioning here that, because of the very low concentration of CIPRO, the voltammetric profile generated broad peaks where the maximum was almost indistinguishable, which in turn, does not allow its accurately measurement. Accordingly, to overcome this shortcoming, it was used the mathematical processing via baseline-corrected second-order derivatives. The approach successful help to detect a well-defined peak current that can be translated into quantitative information, even at the tracelevel. Hence, the results proved that the featured device combined with mathematical processing works properly and can be useful for further practical trace-level applications in electroanalysis and this issue was studied in the next section.

\subsection{Analytical figures of merit and sample analysis}

Using the optimised condition in Tables S-1 and S-5 and Fig. S4 (Supplementary material), the effect of the ChCl:MA (1:1, molar ratio) mass as an extractor solvent was also evaluated to determine 

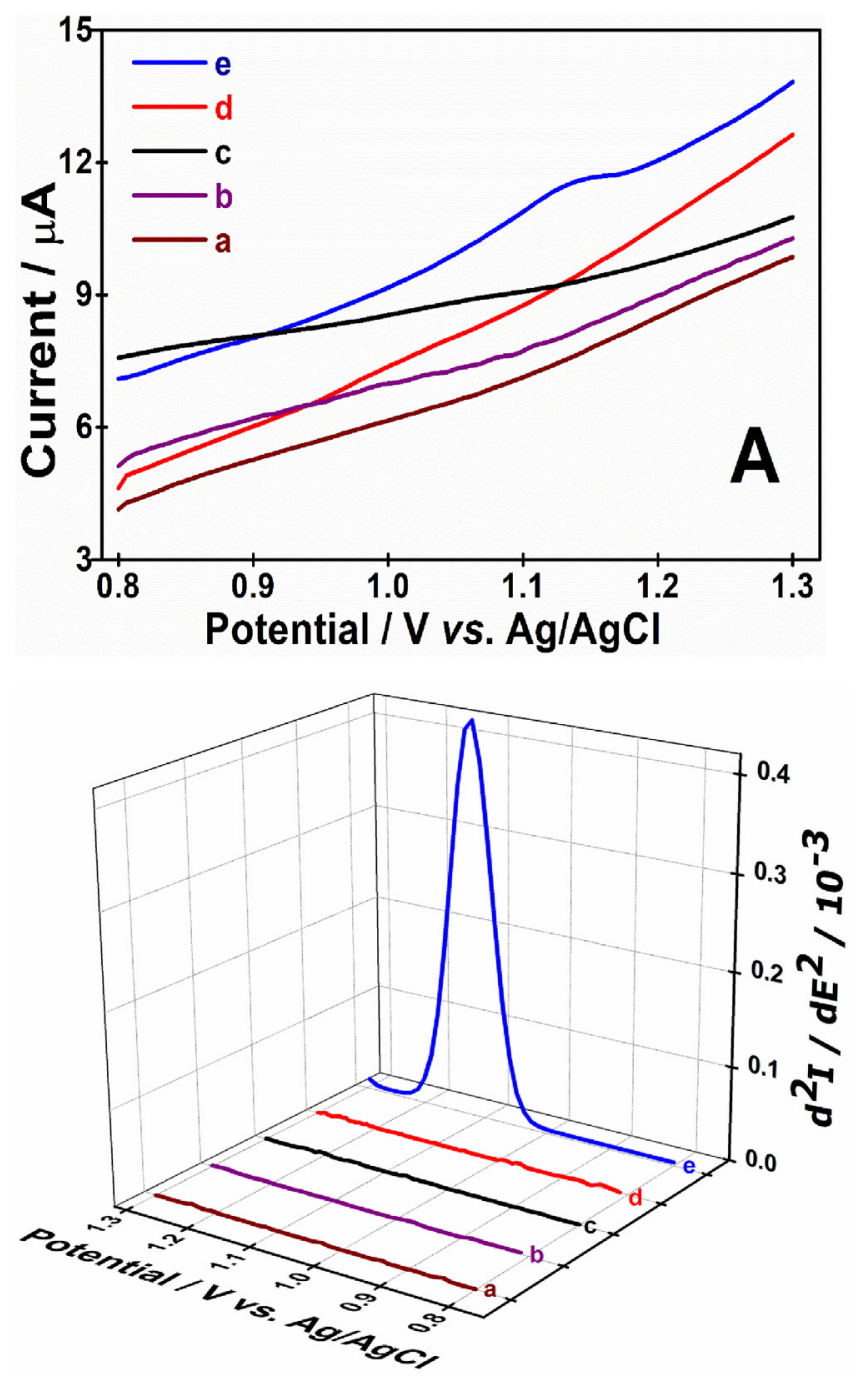

Fig. 4. (A) Original DP voltammograms and (B) baseline-corrected second-order derivative voltammograms registered for CIPRO at $10.0 \mathrm{nmol} \mathrm{L}^{-1}$. (a) Blank: B-R buffer solution prepared in tap water sample, (b) direct detection of CIPRO at $10.0 \mathrm{nmol} \mathrm{L}^{-1}$ in spiked tap water sample, (c) detection after electrochemical pre-concentration (at $0.4 \mathrm{~V}$ for $200 \mathrm{~s}$ ) of the sample as listed in curve b, (d) blank solution as in curve a after pre-concentration using $120 \mathrm{mg}$ of ChCl:MA (1:1, molar ratio) and a proposed device; finally, (e) pre-concentration via the proposed device using $120 \mathrm{mg}$ of $\mathrm{ChCl}$ :MA (1:1, molar ratio) as an extractor solvent to extract CIPRO (at $10.0 \mathrm{nmol} \mathrm{L}^{-1}$ ) from spiked tap water samples. Experimental and instrumental conditions were optimised in Tables S1-S4 and Fig. S-4 (Supplementary material). Extractor: ChCl:MA (1:1, molar ratio).

CIPRO at concentration level of 5.00 and $10.0 \mathrm{nmol} \mathrm{L}^{-1}$, spiked into tap water samples (Fig. 5). The decantation of the extractor: $\mathrm{ChCl}$ :MA (a high density solvent) after pre-concentration was a key step and their influence was obtained by means of the recovery values. In that case, the high density $\mathrm{ChCl}$ :MA (1:1, molar ratio) phase was subjected to mechanical stirring followed by the ice water circulation process in the new configuration of electrochemical cell (Fig. 1). Here, after stopping the process, the enriched phase containing the analyte was decanted on the electrode surface (located at the bottom of the cell, Fig. 1d) to be electrochemically analysed. In addition to this effect, the contact between the extractor solvent and aqueous phase was improved by mechanical stirring during $5.0 \mathrm{~min}$ at $700 \mathrm{rpm}$ (Table S-5, Supplementary material), which was enough to transfer the CIPRO into the ChCl:MA (1:1, molar ratio) phase. To estimate the percentage of

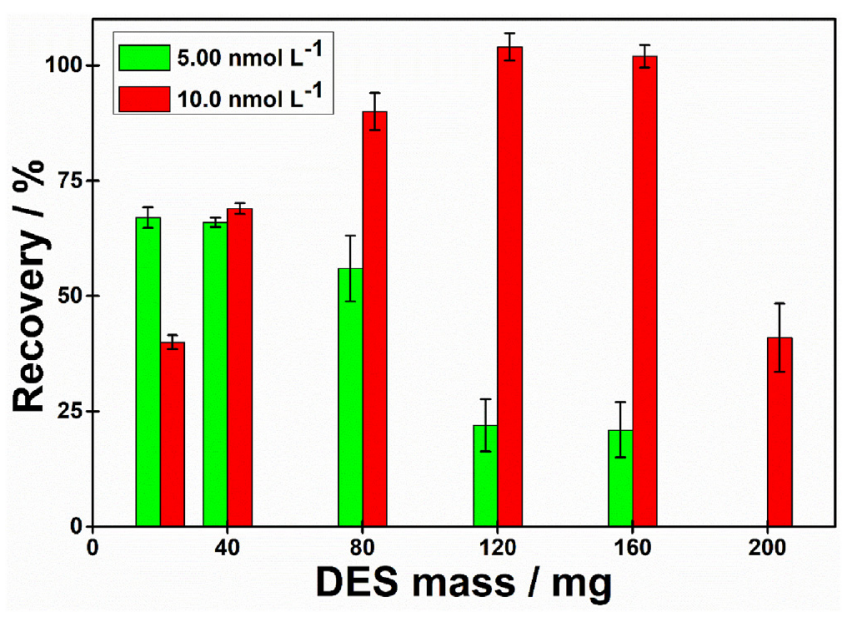

Fig. 5. Recovery test performed to assess the accuracy of the proposed method during determination of CIPRO in spiked tap water samples at two level of concentration. Extractor: ChCl:MA (1:1, molar ratio).

relative recovery (\%RR) of CIPRO shown in Fig. 5, it was used the following equation:

$\% \mathbf{R R}=\frac{\mathbf{I}_{\mathbf{T W}}}{\mathbf{I}_{\mathbf{U W}}} \times 100$

Here, the term $\boldsymbol{I}_{\mathbf{T W}}$ is the measured peak current intensity (duplicate measurements) for the spiked tap water sample, and $\boldsymbol{I}_{\boldsymbol{U} \boldsymbol{W}}$ is the measured peak current intensity (replicate measurements) for the spiked ultra-pure water sample at the same concentration level. The obtained RR values summarised in Fig. 5 indicate that the highest extraction efficiency could be achieved with the ChCl:MA (1:1, molar ratio) mass ranged between 80 and $160 \mathrm{mg}(R R=90 \pm 4.0 ; 104 \pm 3.0$ and $102 \pm 2.5)$ for the spiked level at $10.0 \mathrm{nmol} \mathrm{L}^{-1}$. It is worth to note that these results are in agreement with those obtained in Fig. S-8 (Supplementary material), in which the efficiency of extraction process (to assess the main variable effects in Doehlert matrix, Table S-4 and Fig. S-8, (Supplementary material) measured by RR (spiked level tap water at $10.0 \mathrm{nmol} \mathrm{L}^{-1}$ ) also have maximum values ranged between 80 and $120 \mathrm{mg}$ of ChCl:MA ( $1: 1$, molar ratio) mass. Whereas, when the spiked level was lowered to $5.00 \mathrm{nmol} \mathrm{L}^{-1}$, the RR values were decreased to $67 \pm 2.3,66 \pm 1.1$ and $56 \pm 7.1 \%$ for the ChCl:MA $(1: 1$, molar ratio) mass of 20,40 and $80 \mathrm{mg}$, respectively. These low RR values, at concentration level $5.00 \mathrm{nmol} \mathrm{L}^{-1}$, may not be related to matrix effect, since there is no interference peak (from target tap water sample) that overlapped with the CIPRO peak as showed in Fig. 4 (voltammogram d).

Fig. 5 also shows that the \%RR values tend to decrease for both spiked level of concentration (more pronounced at the $\left.5.00 \mathrm{nmol} \mathrm{L}^{-1}\right)$ when the $\mathrm{ChCl}$ :MA (1:1, molar ratio) mass increase above $120 \mathrm{mg}$. To understand this phenomenon, it may be necessary to bring out two concepts behind the DES and its effect as extractor solvent; their viscosity and its interaction with the analytes. The high viscosity of pure DES has been addressed as one of the most recognized disadvantage, leading to a slow mass transfer during the extraction process [30,31]. To overcome this problem, the processes include the dilution with methanol, acetonitrile or a small amount of water to reduce their viscosity and improve the mass transfer into the DES phase. Regarding the general mechanisms involved in the interaction between DES and the analytes, the insights that take places may be more complex because of the importance of geometric and energy parameters [30-34]. In some 
cases, it should be considered the functional groups and/or the steric hindrances around the structures involved in this process. For example, it was previously reported that the DES having carboxyl groups enable more strong interaction with polyphenols groups than such having only hydroxyl groups [30,31]. Also, literature survey shows that many claimed mechanisms involved into the DES, used either as solvent in organic reactions as in catalysis process, occurs through hydrogen bonds $[32,33]$.

Taking in account the aforesaid studies, thus, we understand that the general mechanism for the interaction ChCl:MA and CIPRO involve the stronger interactions (e.g., $\mathrm{O}-\mathrm{H}-----\mathrm{N}$, $\mathrm{N}-\mathrm{H}-----\mathrm{O}$ and $\mathrm{O}-\mathrm{H}-----\mathrm{O}$ ), due to the greater availability of the electron pairs of these donor atoms, as shown in some scientific reports [30-34]. In this case, we ignore weaker interactions, such as interactions with the fluorine atom as well as the interactions that apparently would be very far. The proposed mechanism in Fig. 6 (representation in two-and three-dimensional array), shows that CIPRO was designed favoring intramolecular hydrogen bonding between carboxylic acid and ketone functions. The piperazinyl substituent was also designed in chair conformation because of its great stability. In the propose mechanism, malonic acid species (from ChCl:MA) interact with CIPRO by either as donors as acceptors of hydrogen bonds. In this pathway, one function carboxylic acid (from malonic acid) acts as a hydrogen bonding donor $(\mathrm{O}-\mathrm{H})$ by interaction with the nitrogen atom from piperazinyl group and as acceptor by interaction between the oxygen atom (from carbonyl group) with the $\mathrm{N}-\mathrm{H}$ from piperazinyl (donor). The second function carboxylic acid (from malonic acid) acts as a donor $(\mathrm{O}-\mathrm{H})$ by interacting with the oxygen atom from quinolinone (CIPRO acting as acceptor). From Fig. 6, it can see that the CIPRO also acts as a donor ( $\mathrm{O}-\mathrm{H}$ from carboxylic acid group) and malonic acid as an acceptor (oxygen atom from carbonyl group). Finally, we believe that the hydrogen bonding network may have directly influenced in the process of CIPRO-extraction from tap water samples. However, when the mass of ChCl:MA (1:1, molar ratio) become greater than $160 \mathrm{mg}$, its high viscosity may not allow the transfer of CIPRO (from aqueous phase) to the DES phase. This issue, in turn, give rise to a dense DES layer deposited on the working electrode surface that can partially blocks its activity and hinder the electron transfer. Thus, it can be understood that the interaction occurs in amount of ChCl:MA (1:1, molar ratio) enough to meet with the proposed mechanism (Fig. 6) and, any excess must be avoided to ensure the best efficiency of target proposed procedure.

Taking in account the data from Fig. 5, on which the ChCl:MA (1:1, molar ratio) at amount higher than $160 \mathrm{mg}$ tend to trouble the electrochemical response, an analytical curve was obtained after performing one extraction for each concentration level (with replicate of measurement). Fig. 7 shows the baseline-corrected second-order derivatives voltammograms recorded for CIPRO at concentration range between 5.00 and $30.0 \mathrm{nmol} \mathrm{L}^{-1}$ (for only the linear range) into the spiked tap water sample. In the target condition, Fig. 7 (inset) shows the analytical curve (at all studied concentration level 5.00 and $40.0 \mathrm{nmol} \mathrm{L}^{-1}$ ) obtained under the optimised extraction conditions in order to obtain more realistic figures of merit. It could be seen that there is no significant matrix effect that compromises the accurate peak current measurement and the quantitative detection of CIPRO can be appropriately performed. The new calibration curve was linear only at the range of 5.00 and $30.0 \mathrm{nmol} \mathrm{L}^{-1}$ with the coefficient of determination $\left(\mathrm{R}^{2}\right)$ higher than $0.97(n=4)$. The linear range has the follow equation: $\left(\mathrm{D}^{2} \mathrm{I} / \mathrm{DE}^{2}\right) / 10^{-3}=0.046+0.010^{*} \mathrm{C}_{\mathrm{CIPRO}(\mathrm{nM})}$. To validate the proposed approach, the used parameters were: linear range (LR), limit of detection (LoD) and preconcentration factor (PF) as presented in Table 1 as well as the recovery values further presented in Table 2. To estimate the LoD (Table 1), a low concentration of the spiked tap water sample that produces a signal (after extraction) at least three-times great than de noise (sample in absence of analyte) was selected as the lowest concentration of detection. From Table 1, the LoD was estimated as $2.50 \mathrm{nmol} \mathrm{L}^{-1}\left(0.83 \mu \mathrm{g} \mathrm{L}^{-1}\right)$. The preconcentration factor (PF) showed in Table 1 was $35 \pm 1.5$ and was achieved using the following equation:

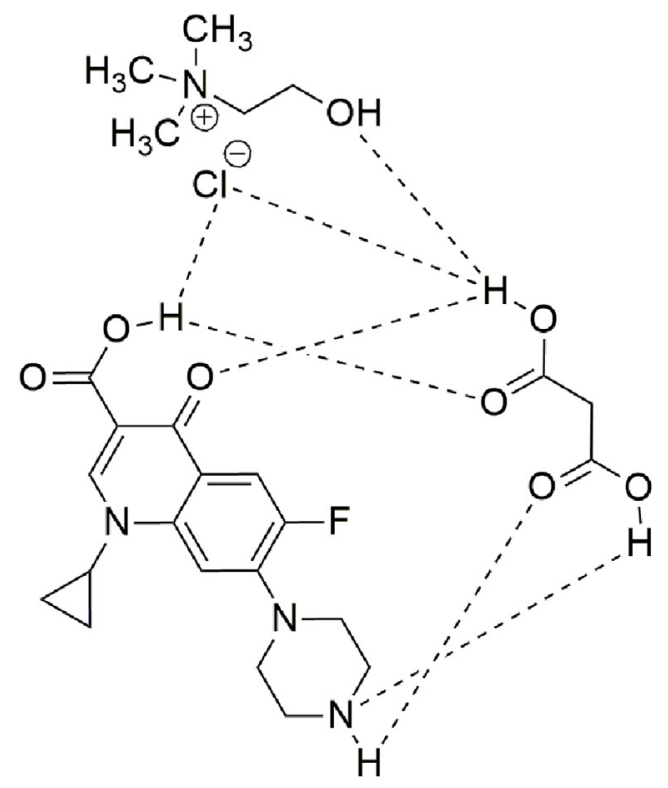

(I)

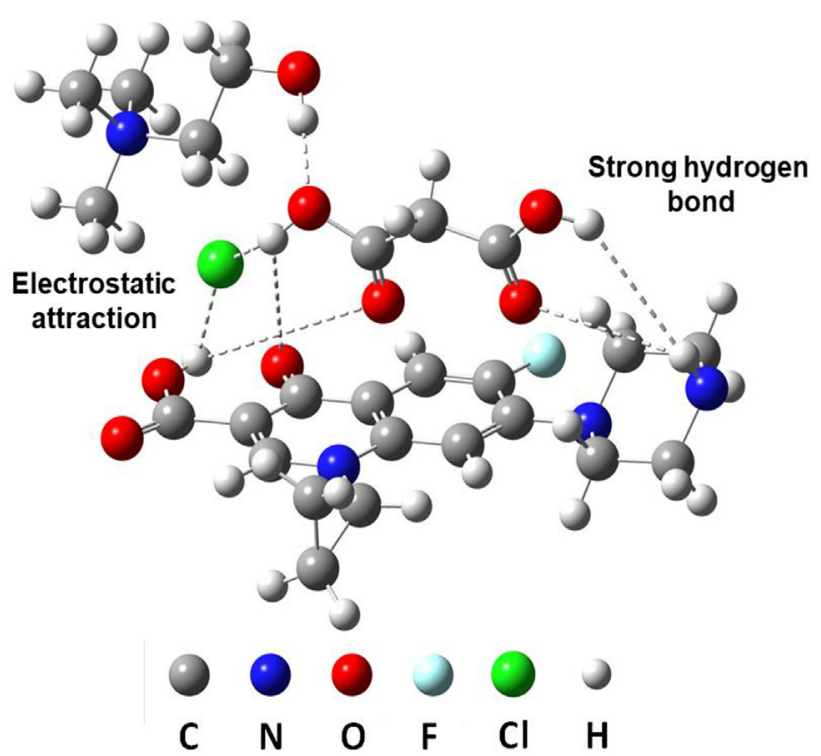

(II)

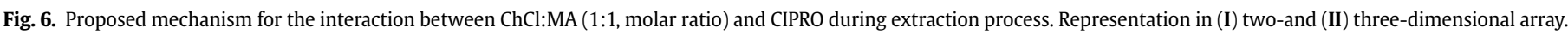




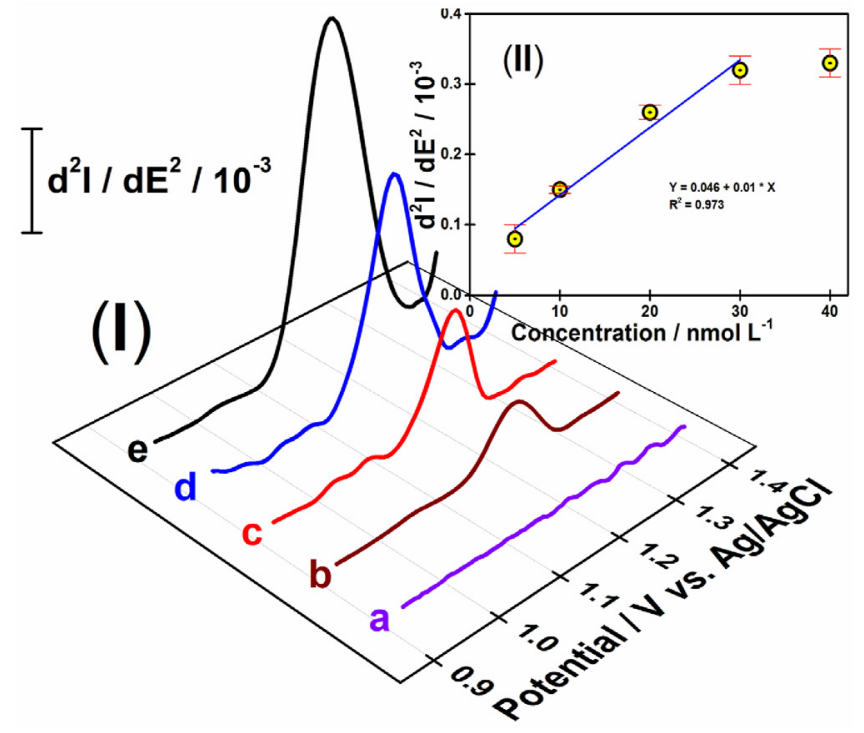

Fig. 7. (I) Baseline-corrected second-order derivative DP voltammograms registered for: (a) Blank: B-R buffer solution prepared in a tap water sample and preconcentration using $120 \mathrm{mg}$ of $\mathrm{ChCl}: \mathrm{MA}$, (b) pre-concentration of CIPRO (at $5.00 \mathrm{nmol} \mathrm{L}^{-1}$ ) from spiked tap water sample using $40 \mathrm{mg}$ of $\mathrm{ChCl}: \mathrm{MA}$, (c) preconcentration of CIPRO (at $10.0 \mathrm{nmol} \mathrm{L}^{-1}$ ) from spiked tap water sample using $120 \mathrm{mg}$ of ChCl:MA, (d) pre-concentration of CIPRO (at $20.0 \mathrm{nmol} \mathrm{L}^{-1}$ ) from spiked tap water sample using $120 \mathrm{mg}$ of $\mathrm{ChCl}: \mathrm{MA}$ and (d) pre-concentration of CIPRO (at $30.0 \mathrm{nmol} \mathrm{L}^{-1}$ ) from spiked tap water sample using $120 \mathrm{mg}$ of $\mathrm{ChCl}$ :MA. (II) Analytical calibration curve for the dependence of the peak current and concentration of CIPRO. Other conditions as in Tables S- 1 and S- 4 as well as Fig. S-4.
$\mathbf{P F}=\frac{\mathbf{C}_{\mathrm{DES} \text { phase }}}{\mathbf{C}_{\text {Sample pahse }}}$

Here, the $\mathbf{C}_{\mathrm{DES}}$ phase is the preconcentrated concentration of the CIPRO in the ChCl:MA (at 1:1, molar ratio) phase and $\mathbf{C}_{\text {Sample pahse }}$ is the initial concentration of the CIPRO in the spiked tap water samples. Table 1 also shows a characteristics of previously reported methods (electrochemical, chromatographic and fluorescence techniques) used here for comparative purposes. From displayed data in Table 1, it can be seen that the proposed method is simpler, enabling fast trace-level determination of CIPRO without complicated sample pretreatment steps.

To demonstrate the applicability, Table 2 summarise the recovery values obtained to assess the accuracy of the proposed method during extraction and determination of CIPRO in two spiked water samples. Fixing the $\mathrm{ChCl}$ :MA (1:1, molar ratio) mass at $40 \mathrm{mg}$ (for the concentration level of $5.00 \mathrm{nmol} \mathrm{L}^{-1}$ ) and $120 \mathrm{mg}$ (for the concentration level of $10.0 \mathrm{nmol} \mathrm{L}^{-1}$ ), a comparative study was performed analysing the tap water and ultrapure water samples spiked at these two level of concentration. The quantification of CIPRO was based on the analytical figures of merit obtained with both external calibration curves: Fig. S-5 (Supplementary material) and Fig. 7. After triplicate measurements, the average recovery values reveal an acceptable accuracy of proposed device to quantify target CIPRO in reported samples. Taking into consideration the evaluated performance of the proposed device, these previous studies are highly promising and are in agreement with our goal of narrowing the interface between electroanalysis and sample preparation in order to pre-concentrate organic analytes and reach the quantitation at trace-level. In addition, the proposed

Table 1

Analytical figures of merit to assess the validation of the proposed method, comparing its performance with previous reported methods.

\begin{tabular}{|c|c|c|c|c|c|c|c|}
\hline Technique/Detector & Extractor & Linear range $\left(\mu \mathrm{g} \mathrm{L}^{-1}\right)$ & $\operatorname{LoD}\left(\mu \mathrm{g} \mathrm{L}^{-1}\right)$ & Spiking $\left(\mu \mathrm{g} \mathrm{L}^{-1}\right)$ & Recovery $(\%) \pm$ RSD & $\mathrm{PF}$ & Ref. \\
\hline DLLME-HPLC-DAD & $\mathrm{H}_{2} \mathrm{O} / \mathrm{ACN}$ & $20-1000$ & 5.61 & $\begin{array}{l}53^{a} \\
400^{a}\end{array}$ & $\begin{array}{l}89 \pm 19 \\
108 \pm 7.0\end{array}$ & - & [35] \\
\hline Fluorescence detection & $\mathrm{Ad} / \mathrm{Tb} \mathrm{CPNPs}$ & $20-4640$ & 20.0 & $\begin{array}{l}994^{\mathrm{b}} \\
1998^{\mathrm{b}}\end{array}$ & $\begin{array}{l}103 \pm 0.1 \\
99 \pm 0.8\end{array}$ & - & [36] \\
\hline Porous-Nafion-MWCNT/BDD & MWCNTs & $16.6-3313$ & $1.66^{\mathrm{c}}$ & - & - & - & [37] \\
\hline Poly- $\beta$-cyclodextrin and l-arginine/CPE & - & $16.6-33134$ & 3.31 & $6626^{\mathrm{b}}$ & $103 \pm 2.4$ & - & [38] \\
\hline This work & DES & $1.66-9.94$ & 0.83 & $\begin{array}{l}1.66 \\
5.75\end{array}$ & $\begin{array}{l}90 \pm 4.3 \\
80 \pm 10\end{array}$ & $35 \pm 1.5$ & This work \\
\hline
\end{tabular}

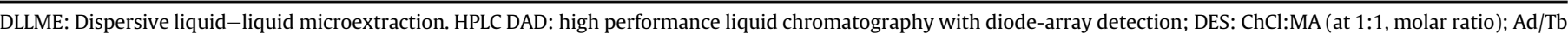

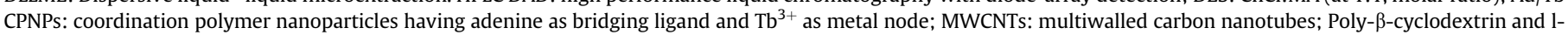
arginine/CPE: polymerization of $\beta$-cyclodextrin and l-arginine modified carbon paste electrode.

a Mineral water as sample.

b Tablets diluted in ultrapure water

c Wastewater effluent; RSD: relative standard deviation; PF: preconcentration factor.

Table 2

Recovery assay to assess the accuracy of the proposed method during the determination of CIPRO in two spiked water samples.

\begin{tabular}{|c|c|c|c|c|}
\hline Sample & Added $\left(\mathrm{nmol} \mathrm{L}^{-1}\right)$ & Found ${ }^{\mathrm{a}}\left(\mathrm{nmol} \mathrm{L}^{-1}\right)$ & Recovery (\%) & RSD (\%) \\
\hline \multirow[t]{4}{*}{ Tap water } & 5.00 & $4.00^{\mathrm{b}}$ & 80.0 & 4.50 \\
\hline & & $4.25^{c}$ & 85.0 & 3.60 \\
\hline & 10.0 & $7.80^{\mathrm{b}}$ & 78.0 & 3.50 \\
\hline & & $8.35^{c}$ & 83.5 & 2.50 \\
\hline \multirow[t]{4}{*}{ Ultrapure water } & 5.00 & $4.35^{\mathrm{b}}$ & 87.0 & 6.15 \\
\hline & & $4.65^{c}$ & 93.0 & 4.50 \\
\hline & 10.0 & $7.10^{\mathrm{b}}$ & 71.0 & 6.60 \\
\hline & & $8.75^{c}$ & 87.5 & 4.25 \\
\hline
\end{tabular}

\footnotetext{
a Average of triplicate measurements.

b Based on the external calibration curve (Fig. S-5, Supplementary material).

c Based on the external calibration curve (Fig. 7); RSD: relative standard deviation.
} 
electrochemical device allowed the traditional vortex/ultrasonication and/or centrifugation steps to be replaced by one simple and in situ mechanical stirring step. Likewise, we have replaced the traditional ice bath and/or further heating process with an adapted jacketed electrochemical cell where the ice water circulates, reducing the process that takes up to three steps in traditional methods, into just one step.

\section{Conclusion}

In this study, it was showed that the new electrochemical cell configuration allowed combining the extraction and preconcentration of CIPRO from very dilute tap water samples prior to electroanalysis at trace-level as a small drop that was directly decanted onto the GCE surface. In addition, the efficiency of ChCl:MA (1:1, molar ratio) and its environmentally friendly characteristics make this DES a promising solvent for use in electroanalysis. The new analytical approach is promising and is expected to deepen the knowledge on the interface between the sample preparation and electroanalysis as well as open new possibilities for further electroanalytical trace-level applications in more environmental interest samples.

\section{Author contributions}

The manuscript was written through contributions of all authors. All authors have given approval to the final version of the manuscript.

\section{Notes}

The authors declare no competing financial interest.

\section{Acknowledgements}

The authors gratefully acknowledge the financial support provided by the FUNDECT/MS and Brazilian funding agency CNPq (grant number: 23/200.258/2014), CNPq/INCT (grant number: 465571/2014-0) and Brazilian Federal Agency for the Improvement of Higher Education - CAPES (grant number: 88881.119436/201601).

\section{Appendix A. Supplementary data}

Supplementary data related to this article can be found at https://doi.org/10.1016/j.electacta.2018.04.134.

\section{References}

[1] J.M. Kokosa, Recent trends in using single-drop microextraction and related techniques in green analytical methods, TrAC Trends Anal. Chem. 71 (2015) 194-204, https://doi.org/10.1016/j.trac.2015.04.019.

[2] M.M.P. Vázquez, P.P. Vázquez, M.M. Galera, M.D.D.G. García, Determination of eight fluoroquinolones in groundwater samples with ultrasound-assisted ionic liquid dispersive liquid-liquid microextraction prior to highperformance liquid chromatography and fluorescence detection, Anal. Chim. Acta 748 (2012) 20-27, https://doi.org/10.1016/j.aca.2012.08.042.

[3] C.H. Xu, G.S. Chen, Z.H. Xiong, Y.X. Fan, X.C. Wang, Y. Liu, Applications of solidphase microextraction in food analysis, TrAC Trends Anal. Chem. 80 (2016) 12-29, https://doi.org/10.1016/j.trac.2016.02.022.

[4] M. Rezaee, Y. Assadi, M.-R. Milani Hosseini, E. Aghaee, F. Ahmadi, S. Berijani, Determination of organic compounds in water using dispersive liquid-liquid microextraction, J. Chromatogr. A 1116 (2006) 1-9, https://doi.org/10.1016/ j.chroma.2006.03.007.

[5] E. Fernández, L. Vidal, J. Iniesta, J.P. Metters, C.E. Banks, A. Canals, Screenprinted electrode-based electrochemical detector coupled with in-situ ionicliquid-assisted dispersive liquid-liquid microextraction for determination of 2,4,6-trinitrotoluene, Anal. Bioanal. Chem. 406 (2014) 2197-2204, https:// doi.org/10.1007/s00216-013-7415-y.

[6] C. Almécija, A. Cobelo-García, J. Santos-Echeandía, Improvement of the ultra- trace voltammetric determination of $\mathrm{Rh}$ in environmental samples using signal transformation, Talanta 146 (2016) 737-743, https://doi.org/10.1016 j.talanta.2015.06.032.

[7] A. Ballesteros-Gómez, S. Rubio, Recent advances in environmental analysis, Anal. Chem. 83 (2011) 4579-4613, https://doi.org/10.1021/ac200921j.

[8] A.P. Abbott, D. Boothby, G. Capper, D.L. Davies, R.K. Rasheed, Deep eutectic solvents formed between choline chloride and carboxylic Acids: versatile alternatives to ionic liquids, J. Am. Chem. Soc. 126 (2004) 9142-9147, https:// doi.org/10.1021/ja048266j.

[9] B.-Y. Zhao, P. Xu, F.-X. Yang, H. Wu, M.-H. Zong, W.-Y. Lou, Biocompatible deep eutectic solvents based on choline chloride: characterization and application to the extraction of rutin from Sophora japonica, ACS Sustain. Chem. Eng. 3 (2015) 2746-2755.

[10] T. Tan, Z. Li, X. Mao, Y. Wan, H. Qiu, Deep eutectic solvent-based liquid-phase microextraction for detection of plant growth regulators in edible vegetable oils, Anal. Methods 8 (2016) 3511-3516.

[11] E.L. Smith, A.P. Abbott, K.S. Ryder, Deep eutectic solvents (DESs) and their applications, Chem. Rev. 114 (2014) 11060-11082, https://doi.org/10.1021/ cr300162p.

[12] F. Scholz, Voltammetric techniques of analysis: the essentials, ChemTexts 1 (2015) 17.

[13] L.M. Moretto, K. Kalcher, Environmental Analysis by Electrochemical Sensors and Biosensors, Springer, New York, New York, NY, 2014, https://doi.org/ 10.1007/978-1-4939-0676-5.

[14] A. Cobelo-García, J. Santos-Echeandía, D.E. López-Sánchez, C. Almécija D. Omanović, Improving the voltammetric quantification of ill-defined peaks using second derivative signal transformation: example of the determination of platinum in water and sediments, Anal. Chem. 86 (2014) 2308-2313, https://doi.org/10.1021/ac403558y.

15] V. Andruch, M. Burdel, L. Kocúrová, J. Šandrejová, I.S. Balogh, Application of ultrasonic irradiation and vortex agitation in solvent microextraction, TrAC Trends Anal. Chem. 49 (2013) 1-19, https://doi.org/10.1016/ j.trac.2013.02.006.

[16] M. Petrovic, Methodological challenges of multi-residue analysis of pharmaceuticals in environmental samples, Trends Environ. Anal. Chem. 1 (2014) e25-e33, https://doi.org/10.1016/j.teac.2013.11.004.

[17] N. Bunyakul, A.J. Baeumner, Combining electrochemical sensors with miniaturized sample preparation for rapid detection in clinical samples, Sensors 15 (2014) 547-564.

[18] A.J. Bard, L.R. Faulkner, J. Leddy, C.G. Zoski, Electrochemical Methods: Fundamentals and Applications, Wiley, New York, 1980.

[19] J. Wang, S. Bollo, J.L. Lopez Paz, E. Sahlin, B. Mukherjee, Ultratrace measurements of nucleic acids by baseline-corrected adsorptive stripping squarewave voltammetry, Anal. Chem. 71 (1999) 1910-1913, https://doi.org/ 10.1021/ac981432j.

[20] J.W. Fuquay, P.F. Fox, P.L.H. McSweeney, Encyclopedia of Dairy Sciences, Academic Press, 2011.

[21] M. Stoytcheva, R. Zlatev, V. Gochev, Z. Velkova, G. Montero, M.T. Beleño, Amperometric biosensors precision improvement. Application to phenolic pollutants determination, Electrochim. Acta 147 (2014) 25-30, https:/| doi.org/10.1016/J.ELECTACTA.2014.09.106.

[22] L.H. De Oliveira, M.A.G. Trindade, Baseline-corrected second-order derivative electroanalysis combined with ultrasound-assisted liquid-liquid microextraction: simultaneous quantification of fluoroquinolones at low levels, Anal. Chem. 88 (2016) 6554-6562, https://doi.org/10.1021/ acs.analchem.6b01379.

[23] U. Bilibio, L.H. de Oliveira, V.S. Ferreira, M.A.G. Trindade, Enhanced simultaneous electroanalytical determination of two fluoroquinolones by using surfactant media and a peak deconvolution procedure, Microchem. J. 116 (2014) 47-54, https://doi.org/10.1016/j.microc.2014.04.009.

[24] L.H. de Oliveira, L.A. Pradela, A.L. Santos, R.M. Takeuchi, M.A.G. Trindade, Mathematical processing comparison of voltammetric data: application with the simultaneous determination of fluoroquinolones, Quim. Nova 38 (2015) 1300-1306.

[25] B. Petrie, R. Barden, B. Kasprzyk-Hordern, A review on emerging contaminants in wastewaters and the environment: current knowledge, understudied areas and recommendations for future monitoring, Water Res. 72 (2014) 3-27, https://doi.org/10.1016/j.watres.2014.08.053.

26] A. Jia, Y. Wan, Y. Xiao, J. Hu, Occurrence and fate of quinolone and fluoroquinolone antibiotics in a municipal sewage treatment plant, Water Res. 46 (2012) 387-394, https://doi.org/10.1016/J.WATRES.2011.10.055.

27] L. Zhu, B. Santiago-Schübel, H. Xiao, H. Hollert, S. Kueppers, Electrochemica oxidation of fluoroquinolone antibiotics: mechanism, residual antibacteria activity and toxicity change, Water Res. 102 (2016) 52-62, https://doi.org/ 10.1016/j.watres.2016.06.005.

[28] R.F. Teófilo, M.M.C. Ferreira, Chemometrics II: spreadsheets for experimental design calculations, a tutorial, New Chem. 29 (2006) 338-350, https://doi.org/ 10.1590/S0100-40422006000200026.

[29] L.M. Alencar, L.H. de Oliveira, R. Backes, T.M. Rosa, P.R. de Oliveira, R.A.B. da Silva, M.A.G. Trindade, Liquid-liquid extraction coupled to batch injection analysis for electroanalysis of levofloxacin at low concentration level, Electroanalysis 29 (2017) 2559-2564, https://doi.org/10.1002/elan.201700364.

[30] N. Fu, R. Lv, Z. Guo, Y. Guo, X. You, B. Tang, D. Han, H. Yan, K.H. Row, Environmentally friendly and non-polluting solvent pretreatment of palm samples for polyphenol analysis using choline chloride deep eutectic solvents, 
J. Chromatogr. A 1492 (2017) 1-11, https://doi.org/10.1016/ J.CHROMA.2017.02.036.

[31] Y. Dai, G.-J. Witkamp, R. Verpoorte, Y.H. Choi, Tailoring properties of natural deep eutectic solvents with water to facilitate their applications, Food Chem. 187 (2015) 14-19, https://doi.org/10.1016/J.FOODCHEM.2015.03.123.

[32] M.A.P. Martins, G.C. Paveglio, L.V. Rodrigues, C.P. Frizzo, N. Zanatta, H.G. Bonacorso, Promotion of 1,3-dipolar cycloaddition between azides and [small beta]-enaminones by deep eutectic solvents, New J. Chem. 40 (2016) 5989-5992, https://doi.org/10.1039/C5NJ03654B.

[33] H.R. Lobo, B.S. Singh, G.S. Shankarling, Bio-compatible eutectic mixture for multi-component synthesis: a valuable acidic catalyst for synthesis of novel 2,3-dihydroquinazolin-4(1H)-one derivatives, Catal. Commun. 27 (2012) 179-183, https://doi.org/10.1016/J.CATCOM.2012.07.020.

[34] T. Steiner, The hydrogen bond in the solid state, Angew. Chem. Int. Ed. 41 (2002) 48-76.

[35] A.V. Herrera-Herrera, J. Hernández-Borges, T.M. Borges-Miquel, M.Á. Rodríguez-Delgado, Dispersive liquid-liquid microextraction combined with ultra-high performance liquid chromatography for the simultaneous determination of 25 sulfonamide and quinolone antibiotics in water samples, J. Pharmaceut. Biomed. Anal. 75 (2013) 130-137, https://doi.org/10.1016/ j.jpba.2012.11.026.

[36] H. Tan, L. Zhang, C. Ma, Y. Song, F. Xu, S. Chen, L. Wang, Terbium-based coordination polymer nanoparticles for detection of ciprofloxacin in Tablets and biological fluids, ACS Appl. Mater. Interfaces 5 (2013) 11791-11796, https:// doi.org/10.1021/am403442q.

[37] P. Gayen, B.P. Chaplin, Selective electrochemical detection of ciprofloxacin with a porous nafion/multiwalled carbon nanotube composite film electrode, ACS Appl. Mater. Interfaces 8 (2016) 1615-1626, https://doi.org/10.1021/ acsami.5b07337.

[38] F. Zhang, S. Gu, Y. Ding, Z. Zhang, L. Li, A novel sensor based on electropolymerization of $\beta$-cyclodextrin and l-arginine on carbon paste electrode for determination of fluoroquinolones, Anal. Chim. Acta 770 (2013) 53-61, https://doi.org/10.1016/J.ACA.2013.01.052. 